\section{Discussion}

Cardiovascular involvement occurs in one fourth of patients with relapsing polychondritis. ${ }^{3}$ Aortic regurgitation is the most common cardiovascular complication and is seen in $4 \%$ to $9 \%$ of patients. ${ }^{4}$ Surgical intervention is rarely necessary for patients with cardiac involvement. Twenty-three such cases have been reported in the literature thus far, and all patients required aortic valve replacement. ${ }^{2}$ Aortic valve replacement alone is associated with high rates of reoperation within the first few years. Lang-Ladzdunski and associates $^{2,3}$ have reported that within 4 years, $23.8 \%$ of those who underwent aortic valve replacement had periprosthetic leak or aneurysm. Most $(52.6 \%)$ of the patients died of a cardiovascular cause in the same period. Inflammation of the aorta and friability caused by the use of steroids are thought to be responsible for surgical failures. A Bentall procedure does not carry a higher surgical risk in these patients and is probably the better option. ${ }^{3}$

Our patient had myocardial infarction caused by progressive coronary ostial stenosis. This is a very rare manifestation, and only one case has been reported thus far. ${ }^{5}$ Disease progression in the absence of use of an immunosuppressant like cyclophosphamide and trauma caused by the use of ostial cardioplegia could have accelerated the inflammatory process, leading to critical stenosis within a short span of 6 months. It is therefore better to use retrograde cardioplegia rather than traumatize the coronary ostia, especially in those in whom the ostia are only mildly involved by the disease process. Although the coronary ostia can be visualized
\end{abstract}

on the table, a preoperative coronary angiogram is essential because it would provide an objective measure of coronary ostial involvement. Thus a concomitant coronary artery bypass graft can be planned well in advance rather than encounter surprises on the table and face a dilemma about whether to graft. If the coronary ostial stenosis is not significant, a Bentall procedure alone would suffice. However, the coronary ostia should not be traumatized during the operation. These patients require close and careful lifelong follow-up to detect the onset of critical coronary ostial stenosis at a stage at which intervention would be beneficial. In this group of patients, steroids and cyclophosphamide can be added postoperatively, although their efficacy in preventing progression or relapses is not universal. ${ }^{4}$

\section{References}

1. Trentham DE, Le CH. Relapsing polychondritis. Ann Intern Med. 1998; 129:114-22.

2. Lang-Lazdunski L, Hvass U, Paillole C, Pansard Y, Langlois. Cardiac valve replacement in relapsing polychondritis. A review. Heart Valve Dis. 1995;4:227-35.

3. Lang-Lazdunski L, Pansard Y, Hvass U. Aortic valve replacement in relapsing polychondritis. J Thorac Cardiovasc Surg. 1997;114:131-2.

4. Selim A-GA, Fulford LG, Mohiaddin RH, Sheppard MN. Active aortitis in relapsing polychondritis. J Clin Pathol. 2001;54:890-2.

5. Yung A, Charleson HA, Ullal R, Doube A. A case of relapsing polychondritis with coronary ostial stenosis and severe aortic incompetence. Semin Arthritis Rheum. 2000;30:144-6.

\title{
Rapidly expanding right coronary artery aneurysm
}

\author{
Paolo Pepino, MD, ${ }^{a}$ Piermario Oliviero, MD, ${ }^{\mathrm{a}}$ Luigi Di Tommaso, ${ }^{\mathrm{b}}$ and Paolo Stassano, MD, ${ }^{\mathrm{b}}$ Castel Volturno \\ and Naples, Italy
}

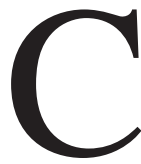

oronary artery aneurysm (CAA) is rare, its etiology is atherosclerotic in the majority of cases, and the right coronary artery (RCA) is the site of predilection. Usually its size remains constant or it enlarges slowly. ${ }^{1}$ Therefore, the presence of a CAA is not always considered to be a surgical indication. ${ }^{2,3}$ Anticoagulation and/or antiaggregation are the recommended treatments.

Rapidly expanding right CAA is different; such an aneurysm may rupture with potentially lethal consequences.

\footnotetext{
From the Department of Cardiothoracic Surgery, Clinica Pineta Grande, Castel Volturno (CE), Italy, ${ }^{a}$ and the Department of Cardiac Surgery, University Federico II, Naples, Italy. ${ }^{\mathrm{b}}$

Received for publication Jan 22, 2006; accepted for publication Jan 30, 2006.

Address for reprints: Paolo Pepino, MD, Via Manzoni 78, 80123, Naples, Italy (E-mail: paolo.pepino@fastwebnet.it).

J Thorac Cardiovasc Surg 2006;131:1396-7

$0022-5223 / \$ 32.00$

Copyright $\odot 2006$ by The American Association for Thoracic Surgery doi:10.1016/j.jtcvs.2006.01.037
}

We describe the case of a successful repair of a rapidly expanding right CAA with prompt surgical correction.

\section{Clinical Summary}

A 50-year-old male patient was admitted to our hospital for coronary angiography in June 2003 . He was completely asymptomatic and he denied any history of inflammatory or connective tissue disease. The electrocardiogram showed an abnormal ST tract. Results of the stress test were negative, but he smoked 40 cigarettes per day, was dyslipidemic and hypertensive, and had a positive family history of ischemic heart disease. Coronary angiography revealed normal coronary arteries but with a slow flow of contrast medium in the coronary bed and an aneurysm (Figure $1, \mathrm{~A})$ of the RCA. The patient was prescribed warfarin (international normalized ratio of 2.5) and was dischared from the hospital.

In May 2004 the patient, still asymptomatic, had a repeat coronary angiogram. There was no coronary stenosis, the flow in the coronary bed was as slow as in the previous year, but the right CAA was significativelly enlarged (Figure 1, B). The rapidity of enlargement of the CAA prompted surgical intervention even in the absence of symptoms. 

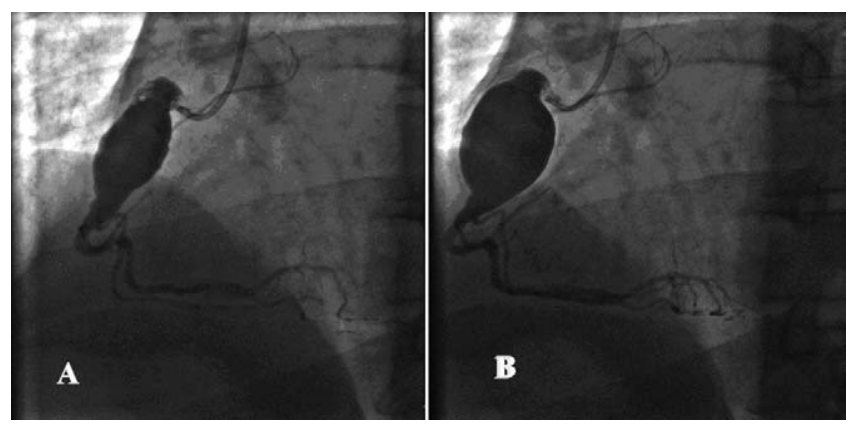

Figure 1. A, Right coronary artery aneurysm at first angiogram. B, Eleven months later.

At surgery, the right CAA was soft on palpation and bluish. To facilitate isolation of the aneurysm from the surrounding myocardium and to preserve the sinus node artery, we preferred to use cardiopulmonary bypass (CPB). During normothermic CPB, the aorta was clamped and myocardial protection was achieved with antegrade and retrograde isothermic potassium cardioplegia. The right CAA was carefully isolated from the surrounding myocardium and was excised. There was no thrombus in the lumen and the wall was very thin. The two stumps of the RCA were oversewn. The proximal tract of the RCA, which gave origin to the sinus node artery, was left intact in situ, thereby preserving antegrade flow into the artery. The right internal thoracic artery (RITA), harvested as a pedicle, was anastomosed end to side to the distal RCA. CPB was interrupted with minimal support (dobutamine $5 \gamma \cdot \mathrm{kg}^{-1} \cdot \mathrm{min}^{-1}$ ), the postoperative course was uneventful, and the patient was discharged home in the fifth postoperative day. Histologic examination of the CAA revealed evidence of atherosclerotic inflammation.

Coronary angiography performed 6 months after the operation showed complete disappearance of the aneurysm with the sinus node artery left in situ (Figure 2, A), a patent anastomosis of the RITA to the distal RCA (Figure 2, B), and slow flow of contrast in the coronary bed.

\section{Discussion}

CAAs are rare, their incidence being $0.2 \%$ to $4.9 \%$ of all coronary disease. ${ }^{1,4}$ Atherosclerosis is the most common cause of CAAs, followed by inflammation, infections, connective tissue disorders, and trauma. The mere presence of a CAA is not a surgical indication, and treatment with anticoagulants and/or antiaggregants is usually recommended. ${ }^{2,3}$

When surgery is indicated, the preferred treatment is resection of the aneurysm and reconstruction of the coronary continuity with
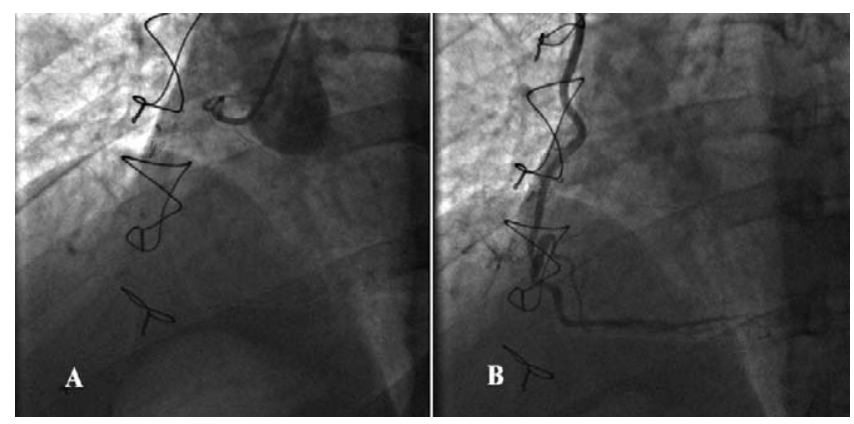

Figure 2. A, Proximal segment of the right coronary artery with the sinus node artery left in situ. B, Patent anastomosis of the right internal thoracic artery to the distal right coronary artery.

vein or arterial grafts is the preferred treatment. The exclusion of the aneurysm may sacrifice important coronary collaterals, such as the sinus node artery, which is important in maintaining normal heart rhythm. In fact, its sacrifice during the transseptal approach to the mitral valve is associated with the occurrence of postoperative supraventricular arrhythmia. ${ }^{5}$ Therefore, in our patient we attempted to preserve the sinus node artery, which continued to receive blood from the orifice of the RCA while the distal segment of the RCA was revascularized with the RITA.

Our case is interesting because of the rapid enlargement of the aneurysm in the course of 1 year, during which the patient remained completely asymptomatic. CAAs rarely are asymptomatic. They usually present with angina, myocardial infarction, and sudden death; furthermore, their size remains constant or they enlarge slowly. From our experience we can conclude that once a CAA is diagnosed, the surgeon must monitor the patient closely and be prepared to intervene before lethal consequences can appear.

\section{References}

1. Moriyama Y, Hisatomi K, Shimokawa S, Taira A, Arima S. Coronary artery aneurysm repaired with saphenous vein patch plasty. Ann Thorac Surg. 1998;65:561-2.

2. Dralle JG, Turner C, Hsu J, Replogle RL. Coronary artery aneurysm after angioplasty and atherectomy. Ann Thorac Surg. 1995;59:1030-5.

3. Tunick PA, Slater J, Kronzon I, Glassman E. Discrete atherosclerotic coronary artery aneurysm: a study of 20 patients. J Am Coll Cardiol. 1990;15:279-82.

4. Swaye PS, Fisher LD, Litwin P, Vignola PA, Judkins MP, Kemp HG, et al. Aneurysmal coronary artry disease. Circulation. 1984;67:134-8.

5. Kanemitsu N, Nakamura T, Okabe M, Tenpaku H, Wariishi S, Ohki A. Giant coronary artery aneurysm arising from the sinus node artery. Ann Thorac Surg. 2001;72:1373-4. 